\title{
TRES RETOS PARA LA ENERGÍA EN ESPAÑA: COMPETITIVIDAD, SEGURIDAD Y CRECIMIENTO
}

\author{
Autores: Eloy Álvarez Pelegry ${ }^{1}$ \\ Director de la Cátedra de Energía de Orkestra - Universidad de Deusto \\ Académico de la Real Academia de Ingeniería
}

\section{Resumen}

El sector energético español se enfrenta a tres retos principales: competitividad (suministro a bajo coste, en especial a los sectores intensivos en energía), seguridad de suministro (vía diversificación de fuentes, y exploración y eventual explotación de recursos domésticos) y crecimiento, tras fuertes inversiones en infraestructuras, en un contexto de estancamiento de la demanda.

Para comprender mejor los retos, se analizan varios drivers o factores de contexto: mercados globales de energía, tendencias de la economía global, descarbonización, revolución del shale gas en EE. UU., Unión de la Energía en la UE,

${ }^{1}$ ealvarezpelegry@orkestra.deusto.es

El autor desea expresar su agradecimiento a Macarena Larrea y a Jaime Menéndez por sus aportaciones al presente documento. 


\title{
Eloy Álvarez Pelegry
}

transición energética en Alemania, generación distribuida y transporte. En base a todo lo anterior se finaliza con unas reflexiones y algunas sugerencias.

Palabras clave: competitividad; seguridad; crecimiento; demanda de energía; inversiones en energía; factores o drivers; mercados domésticos e internacionales; industria.

\section{Three challenges for energy in spain: competitiveness, security and growth}

\begin{abstract}
Nowadays the Spanish energy sector is facing three main challenges: competitiveness (low cost energy especially to energy intensive industries), security of supply (through diversification, and the exploration and eventual production of domestic resources) and growth (in a framework of low energy demand and important investments in energy infrastructures).

In order to better understand energy challenges, several factors or drivers are examined, namely: global energy markets, global economy trends, decarbonization, shale gas revolution in the USA, European Energy Union, the German energy transition, distributed generation and transport. Based on the above the document ends with some thoughts and suggestions.
\end{abstract}

Key Words: competitiveness; security; growth; energy demand; energy investments; energy drivers; domestic and international markets; industry.

\section{INTRODUCCIÓN}

El presente trabajo trata de examinar los retos actuales del sector energético español. Para ello, se analizan los factores que, en el marco de las tendencias globales, están conformando nuevas situaciones y escenarios, que afectan o que tienen implicaciones para los retos identificados.

En este sentido, no se pueden comprender los retos a los que se enfrenta el sector energético del país si no se sitúan las energías primarias (petróleo, gas y carbón) en el contexto global. Por otra parte, no es fácil entender la competitividad y la seguridad de suministro si no se tiene en cuenta la revolución del shale gas en 
Estados Unidos, ni las directivas y los objetivos de la Unión Europea. Asimismo, las tendencias europeas ligadas a la generación distribuida y al autoconsumo, relacionadas con la descarbonización, son también cuestiones fundamentales que afectan a la competitividad y al modo de crecimiento. Además, la evolución de la economía mundial afecta al crecimiento en España tanto en el ámbito económico como en el industrial y en el energético.

Alemania es por varios motivos una referencia en Europa, dado que su proceso de transición energética (Energiewende) presenta numerosas facetas, unas positivas y otras no tanto, que, por un lado, influyen en Europa y, por otro, plantea, temas como el de la competitividad de la industria intensiva en consumo de electricidad, que es de especial interés, para la industria española que trata de competir en el ámbito internacional.

Tampoco se puede obviar el tema de la dependencia de los combustibles fósiles y su relación con el transporte, que ligado a la descarbonización y a la seguridad de suministro plantean cuestiones tanto económicas como geopolíticas, muy relevantes.

Tras el examen de los factores de contexto o drivers se analizan individualmente los retos nacionales, considerando sus implicaciones y sugiriendo algunas ideas.

Los retos, en mi opinión son tres: la competitividad, la seguridad y el crecimiento del propio sector y de la economía, y relacionado con este reto, se consideran dos: la energía como elemento tractor para la reindustrialización y el modo de crecimiento.

Hoy no parece posible pensar en retos, sin tener en cuenta los periodos temporales que requiere su consecución y sin considerar los procesos de transformación, que afectan a otros sectores relacionados con el energético y, en particular, a la industria, por lo que este aspecto, no menor, será objeto de algunas consideraciones. Finalmente se concluye con algunas reflexiones y sugerencias.

\section{EL CONTEXTO DE LOS RETOS ENERGÉTICOS: OCHO TEMAS A TENER EN CUENTA}

El sector energético español no puede considerarse aislado del contexto mundial. Por el contrario es un sector donde los acontecimientos internacionales pueden modificar el panorama existente de una manera muy rápida. Por ello el presente apartado identifica los temas que, se entiende, afectan y conforman o conformarán el entorno al que se enfrentará el sector energético español.

El primero es el papel que desempeñan los mercados globales de energía; el segundo, la ralentización de la economía mundial, acompañada de un crecimiento desigual. El tercer gran tema, que ya ha afectado y a futuro influirá aún más, es la descarbonización de la economía. Un tema, muy relevante para el reto de la competitividad, que afecta a Europa, es la revolución de shale gas en los EE. UU. 


\section{Eloy Álvarez Pelegry}

De nuevo en el ámbito europeo, no se pueden comprender los retos de competitividad, de crecimiento y de búsqueda de la Unión Energética, si no se tiene en cuenta que, desde el punto de vista energético, Europa se encuentra fragmentada. Además, el peso que tiene Alemania en Europa, y su proceso de transición energética (Energiewende), es un factor a considerar muy seriamente, por la relación de la energía, con la industria y el crecimiento, pudiendo ofrecer interesantes lecciones.

Asimismo hay que tener en cuenta dos tendencias, a saber, la generación descentralizada y distribuida y el protagonismo, que progresivamente está teniendo o se desea que tenga, el consumidor individual final. El último tema que se ha de considerar es la importancia que la energía tiene en el transporte junto con los cambios que experimentará en un horizonte de transición.

La tecnología, su evolución y su competitividad, son a buen seguro un tema clave. Sin embargo, en este trabajo, más que identificarlo como un tema específico, se analiza en varios de los factores de contexto, sean estos la descarbonización, la revolución del shale gas, la transición energética en Alemania, la evolución hacia los "prosumidores" y también en el transporte.

\subsection{Los mercados globales de energía}

Es difícil entender el sector energético en su sentido amplio, sin examinar lo que sucede en el mercado del petróleo. El comportamiento de este mercado está influido por los principios de la oferta y la demanda; existiendo además, un grado de relación apreciable entre los precios de las materias primas, la energía y los ciclos económicos.

Por el lado de la demanda, se puede decir que, en los últimos años, ha existido una tendencia a sobreestimarla. Así, la Energy Information Administration de Estados Unidos esperaba para 2015 un consumo global de 103,1 millones de barriles al día; sin embargo, la realidad es un $10 \%$ menor, siendo la demanda en torno a los 92,5 millones ${ }^{2}$. En este sentido, si bien en el año 2000 las expectativas de la demanda eran de crecimiento lineal; hoy se prevé un crecimiento más moderado.

La geopolítica del mercado del crudo también tiene un papel decisivo. De esta manera, la revolución del shale oil en los EE. UU; los incrementos en la producción de gas en países como Irak, y a futuro de Irán; la política de la OPEP y, en particular, de Arabia Saudí, han influido sobre los precios del crudo y conforman las expectativas sobre los mismos.

En el ámbito del gas, es conocido el diferencial de precios entre diversas regiones; Japón y Lejano Oriente, Europa y Norteamérica. Así los precios del gas en Japón

${ }^{2}$ Clemente, J., "Abated Incremental Oil Demand Extends Future Supply", Forbes (abril 2015), disponible en: http://www.forbes.com/sites/judeclemente/2015/04/29/incremental-oil-demand-wont-gounabated/ 
venían siendo cuatro veces superiores a los de EE. UU, diferencia que está disminuyendo; y los de Europa dos veces y media superiores. Aunque estas diferencias se amortiguan conforme los precios del petróleo disminuyen, es posible que siga existiendo una brecha, en particular, entre Europa y EE. UU.; donde los precios se determinan fundamentalmente por la oferta y la demanda en los mercados organizados.

Esta situación ha espoleado el interés en Europa por impulsar los mercados organizados de gas, ya existentes en el centro y el norte de Europa, y el inicio y la implantación en aquellas áreas donde no existen como en la Península Ibérica. Si bien existe en Europa, una tendencia hacia el desacoplamiento de los precios del gas de los del crudo, a través de los mercados organizados de gas natural; sigue habiendo una gran correlación entre ambos precios, y en particular en España. Así, el 42\% de los precios de gas formados en Europa en 2013, lo fueron en base al crudo (oil price escalation); en comparación con el 19\% a nivel global; o la ausencia de este tipo de mecanismos en América del Norte. Sólo resultan comparables Asia (41\%) y Asia Pacífico (57\%) ${ }^{3}$.

En España, la influencia del precio del crudo sobre los precios del gas es muy patente. A estos efectos, si para el caso español se calcula la correlación existente entre los precios del gas importado y del petróleo (Brent), se observaría la estrecha relación existente. Dicha correlación alcanza valores cercanos a 0,96, en caso de que se tomaran los precios del gas importado y los precios del Brent promedio a 9 meses-1.

Por su parte, los precios del carbón siguen jugando también un papel importante. Es más, en Europa y en España, el carbón ha desplazado la generación con gas; a lo que, entre otros motivos ${ }^{4}$, han contribuido los reducidos precios del $\mathrm{CO}_{2}$ Además, la fijación de los precios del carbón de importación se realiza a nivel internacional.

Por todo lo anterior, los mercados internacionales y regionales de petróleo y sus derivados, del gas y del carbón, son referentes obligados para examinar la situación energética.

\subsection{La economía mundial, crecimientos desiguales y volátiles. Población y urbanización crecientes}

En el ámbito económico, las proyecciones de crecimiento global, encuentran hoy perspectivas menos halagüeñas. China disminuye su crecimiento y su influencia en la economía mundial, y el comportamiento de países "emergentes",

\footnotetext{
${ }^{3}$ International Gas Union, IGU, Wholesale Gas Price Survey, 2014 Edition, A global review of price formation mechanisms 2005-2013, 2014. Disponible en: http://www.igu.org/sites/default/files/node-document-field_file/IGU_GasPriceReport\%20_2014_reduced.pdf

${ }^{4}$ En algún caso regulatorios.
} 


\section{Eloy Álvarez Pelegry}

tanto BRIC ${ }^{5}$ como $\mathrm{MINT}^{6}$, considerados como motores del crecimiento a nivel mundial, han generado incertidumbre; lo que ha llevado a la Reserva Federal de Estados Unidos, de momento, a no subir los tipos de interés. Esto afecta no únicamente a la demanda de energía y a su ritmo de crecimiento, sino también en las inversiones en toda la cadena de valor de la industria relacionada con la energía.

Es también destacable el crecimiento desigual, en términos energéticos, entre regiones que se pronostica para los próximos veinticinco años; crecimiento que vendrá, mayoritariamente, de países no-OCDE7 . De éste, el 60\% tendrá lugar en Asia, donde China seguirá siendo el centro de la demanda energética, hasta 2025; año de inflexión en el que India superará a China. Completan los BRIC, Rusia con un crecimiento medio anual del 0,4\% y Brasil, con el 2,1\%. África acumulará el 12\% del crecimiento, mientras que Oriente Medio presentará un $10 \%$ y América Latina un $8 \% 8$.

En este contexto no se debe olvidar el peso que las energías fósiles y los hidrocarburos tienen y seguirán teniendo a nivel mundial. En este sentido, se espera que para 2030, China haya superado a los EE. UU. en consumo de petróleo ${ }^{9}$. En Rusia el consumo de gas seguirá suponiendo más del 50\% del consumo total de energía. Por otro lado, se espera un aumento de la demanda global de carbón, fuertemente impulsado por Asia, donde India se convertirá, en 2025, en el primer consumidor mundial ${ }^{10}$.

La demanda de energía vendrá acompañada de importantes cambios demográficos. Frente a los 7.300 millones de personas actuales, se prevé que la población total alcance los 9.700 millones en 2050. Hasta la mitad de siglo, África protagonizará el crecimiento con más de un 50\% del total (1.300 millones en África frente a 900 millones en Asia). Europa decrecerá de los 738 millones actuales a 707 en $2050^{11}$. Estos crecimientos vendrán acompañados de un mayor desarrollo de la vida en las ciudades. Si en 2014 el 54\% de la población vivía en áreas urbanas (se superó el 50\%, por primera vez, en 2007), en 2050 esta cifra será del 66\%. De nuevo, África y Asia evolucionarán más rápido, pasando a tener un 56\% y 64\%, respectivamente, de población urbana, frente al 40\% y 48\% actual; y se pasará de las 28 "megaciudades" en la actualidad (más de diez millones de habitantes), a 41 en $2050^{12}$.

\footnotetext{
${ }^{5}$ Brasil, Rusia, India y China.

${ }^{6}$ México, Indonesia, Nigeria y Turquía.

${ }^{7} \mathrm{La}$ OCDE contribuirá sólo con un 3\% a la demanda global de energía primaria, siendo destacable el decrecimiento anual de la Unión Europea y Japón (-0,3\% y -0,2\% respectivamente).

${ }^{8}$ Birol, F. y otros, World Energy Outlook, Agencia Internacional de la Energía, 2014.

${ }^{9}$ En el caso americano, en el año 2030 el peso del petróleo será del 27\%.

${ }^{10}$ AIE, World Energy Outlook 2015, 2015.

${ }^{11}$ Naciones Unidas, World Population Prospects. The 2015 Revision. Key findings and Advance Tables, 2015. Disponible en: http://esa.un.org/unpd/wpp/Publications/Files/Key_Findings_WPP_2015.pdf

${ }^{12}$ Naciones Unidas, World Urbanization Prospects. The 2014 Revision. Highlights, 2014. Disponible en: http://esa.un.org/unpd/wup/Highlights/WUP2014-Highlights.pdf
} 


\subsection{La descarbonización}

La lucha contra el cambio climático, no es entendible si no se hace referencia a la Convención Marco de Naciones Unidas sobre el Cambio Climático (CMNUCC) y los órganos que la integran; entre ellos, la Conferencia de las Partes (COP). Un elemento clave fue el Protocolo de Kioto, que junto con las sucesivas conferencias de las partes, ha tratado el objetivo global de mitigación de las emisiones de gases de efecto invernadero (GEI), influyendo en los cambios en el sector energético.

Desde la firma del Protocolo de Kioto, en 1998, que entró en vigor en 2005, han tenido lugar numerosas reuniones de la COP, siendo una de las más conocidas la COP15 (2009) de Copenhague ${ }^{13}$. En diciembre de 2015, tendrá lugar la Cumbre de París COP21, donde el acuerdo China-EE. UU, el papel de la UE y el deseo de Francia de que la Cumbre sea un éxito, plantean grandes expectativas ${ }^{14}$. Es probable, que de la Cumbre no sólo emerjan acuerdos de importancia, sino que, se refuerce también el consenso general sobre la necesidad de una energía y una economía bajas en carbono. Además, hoy es muy relevante la combinación de problemas globales, como los Gases de Efecto Invernadero (GEI), con preocupaciones locales como la reducción de la contaminación en las ciudades.

La Comisión Europea visualiza una energía con prácticamente cero emisiones de GEI; y unos sectores industriales así como la edificación y el transporte, donde las emisiones disminuyen muy sustancialmente; pasándose de 5,9 $\mathrm{GtCO}_{2} \mathrm{e}$ en 1990, a 1,2 Gt $\mathrm{CO}_{2}$ e en 2050; lo que supone una reducción del $80 \%$ de las emisiones de todos los sectores considerados en el Road Map 205015. Para ello, la eficiencia energética, la incorporación creciente de renovables, el mayor peso del gas natural y la sustitución del carbón como energía primaria, son todos ellos aspectos que se pueden identificar con el intento de reducir las emisiones de GEI y, en particular, del $\mathrm{CO}_{2}$

Como se decía al inicio, la tecnología es un tema clave para el sector energético y, en la lucha contra el cambio climático, debería desempeñar un papel fundamental. En este sentido, para la Agencia Internacional de la Energía (AIE) la mayor contribución de las tecnologías debería venir, sobre todo, de la mejora de la eficiencia en el uso final de combustibles y en electricidad (38\% de las reducciones), así como del empleo de energías renovables (30\%); seguido de la captura

\footnotetext{
${ }^{13}$ Una descripción de las diferentes COP, y los resultados más significativos de las mismas, pueden verse en las negociaciones internacionales de cambio climático: hacia París 2015 en Círculo de Empresarios, "Hacia una energía competitiva, sostenible y garantizada", febrero 2015.

${ }^{14}$ Álvarez, E., "Cambio Climático; el camino a París", Expansión, (16 de mayo 2015), p. 47.

${ }^{15}$ European Climate Foundation, ROADMAP 2050. A practical guide to a prosperous, low-carbon Europe. Technical analysis, 2010. Disponible en: http://www.roadmap2050.eu/attachments/files/Volume1_fullreport_PressPack.pdf
} 


\section{Eloy Álvarez Pelegry}

y almacenamiento de $\mathrm{CO}_{2}$ (CCS) y de la energía nuclear (8\%). También destaca que a pesar del progreso continuado ninguna tecnología está en condiciones de cumplir, ella sola, los objetivos.

Respecto a la industria, a lo largo de las próximas décadas serán la eficiencia energética y la innovación en los procesos, los factores más influyentes en la disminución de las emisiones. Cabe señalar que la AIE atribuye a la industria un $21 \%$ de la disminución de emisiones, tras la energía (39\%) y el transporte (20\%), no debiendo desdeñarse la contribución de los edificios $(12 \%)^{16}$.

Cabe decir que la I+D en energía, en los países de la OCDE, se ha mantenido relativamente constante durante los últimos quince años, lo que no es suficiente. Se puede destacar el caso de China, que se enfrenta a grandes retos en el ámbito energético y que se espera se posicione como líder global en I+D en 2019, superando a Estados Unidos ${ }^{17}$.

\subsection{La revolución del shale gas y shale oil en los EE.UU.}

El desarrollo del gas no convencional y en particular el shale gas en los EE. $\mathrm{UU}$, ha supuesto pasar de una producción de 1,5 bcf/día (15,33 bcm/año), en 2000, a unos 35 bcf/día (357,7 bcm/año) en 2014. Como consecuencia la cuota total de producción de gas no convencional en la producción de gas natural del país, ha pasado de representar el 1\% al 40\%.

Como señalan Álvarez, E. y Suárez, C. $(2015)^{18}$, la revolución del shale gas ha tenido consecuencias importantes en los Estados Unidos. Ha incrementado la base de recursos y reservas y ha elevado la producción nacional de petróleo y gas natural, propiciando así una mayor independencia energética del país. Asimismo ha supuesto un desplazamiento del carbón en la generación eléctrica. Además, ha mejorado la competitividad de la industria, como consecuencia de la caída de los precios del gas (especialmente positivo para la industria intensiva en consumo de gas), lo que también ha repercutido positivamente en la economía en general y en el empleo. Así, el desarrollo del shale gas, ha supuesto una revolución, por la evolución de los precios y porque un país importador de gas natural licuado (GNL) ha pasado a expor$\operatorname{tar}^{19}$ (FERC, 2015).

${ }^{16}$ AIE, Energy Technology Perspectives. Mobility innovation to accelerate Climate Action, 2015.

${ }^{17}$ Poponi, D., Energy Technology Perspectives 2015: Mobilising Innovation to Accelerate Climate Action, conferencia de la Agencia Internacional de la Energía en el Ministerio Federal Austriaco de Transporte, Innovación y Tecnologías de Viena, junio 2015.

${ }_{18}$ Álvarez, E. y Suárez, C., El Gas No Convencional: "Shale Gas" Aspectos estratégicos, técnicos, medioambientales y regulatorios, Marcial Pons. Publicación diponible a finales de enero de 2016.

${ }^{19}$ FERC, North-American LNG import/export terminals. Approved, 2015. Disponible en http://www. ferc.gov/industries/gas/indus-act/lng/lng-approved.pdf 
No puede identificarse una causa única de esta revolución. Entre las que se señalan se encuentran, la tecnología, la I+D, la estrategia y las iniciativas empresariales; así como el tipo de recursos geológicos. No menos importante son los aspectos regulatorios y fiscales, como el régimen de derechos y de propiedad de los hidrocarburos.

La situación de los EE. UU. contrasta con la europea. A futuro, se prevén crecimientos de la demanda modestos del 0,6\% ${ }^{20}$, así como una disminución de la producción en Noruega, Holanda y Reino Unido; de tal manera, que para 2020, la producción de Europa-OCDE, pasará de unos 300 bcm en el año 2000, a menos de $250 \mathrm{bcm}$. Es por ello, que un eventual desarrollo de recursos propios, contribuiría a mitigar las importaciones.

\subsection{Una Europa fragmentada, en busca de la Unión Energética}

La fragmentación europea en energía es manifiesta tanto en los mercados de electricidad como en los más incipientes de gas. En este sentido, para avanzar hacia una integración de éstos, es necesario el desarrollo de las infraestructuras, que permitan la comercialización de energía entre países de la Unión, así como el acoplamiento de los precios a ambos lados de las fronteras.

Por el momento, en el ámbito eléctrico se ha avanzado en la Península Ibérica, con la creación del Mercado Eléctrico Ibérico (MIBEL) en 200721. No obstante, es necesaria la inversión en interconexiones con Francia para que la Península deje de ser una isla en electricidad. Por otro lado, existen numerosos hubs de gas en la UE cuyos precios van convergiendo. Sin embargo, de nuevo, en la Península se está pendiente del desarrollo del mercado gasista.

La diversidad de políticas de apoyo a las renovables y el hecho de que los Estados sean soberanos de su mix energético, lleva a una difícil integración, que en la actualidad trata de instrumentarse mediante el amplio "paraguas" de la Unión Energética ${ }^{22}$. Esta Unión se enmarca en una política climática ambiciosa y su objetivo es facilitar a los consumidores domésticos e industriales de la UE una energía, segura, sostenible, competitiva y asequible.

Para ello, la estrategia aspira alcanzar varios aspectos entre los que cabe señalar una economía baja en carbono, con compañías fuertes, innovadoras y competitivas y que los ciudadanos sean el centro de esa Unión de la Energía, aprovechando las nuevas tecnologías para reducir lo que pagan por la energía y participando

${ }^{20}$ AIE, Gas Medium-Term Market Report, 2015.

${ }^{21}$ Álvarez, E. y Larrea, M., Energy policy: European challenges, Spanish answers. Policy paper 106, March 2014. Notre Europe. Jacques Delors Institute. http://www.institutdelors.eu/media/energyeuandspain-alvarez-larrea-ne-jdi-mar14.pdf?pdf $=$ ok

${ }^{22}$ Comisión Europea, Paquete sobre la Unión de la Energía. Estrategia Marco para una Unión de la Energía resiliente con una política climática prospectiva. COM (2015) 80 final. 


\section{Eloy Álvarez Pelegry}

activamente en el mercado, a la vez que se protege a los consumidores vulnerables. Para alcanzar ese objetivo, según el documento de la Comisión, hay que dejar atrás una economía en la que la energía se basa en un enfoque centralizado, guiado por la oferta y que depende de viejas tecnologías y modelos empresariales que no se han puesto al día. Hay que dar poder a los consumidores, continúa la Comunicación de la Comisión, facilitándoles información y la elección, creando flexibilidad, tanto para gestionar la demanda, como la oferta.

La Unión Energética se estructura en torno a cinco dimensiones y quince puntos de acción. Respecto a las primeras, cabe destacar aquí las referentes a la seguridad de suministro, eficiencia energética, descarbonización y competitividad. La Unión Energética incide también sobre algunos temas que, en este trabajo, se destacan, como la mayor transparencia de los precios de la energía, infraestructuras energéticas para la seguridad e integración de mercados. En el transporte se hace referencia a un mayor desarrollo de combustibles alternativos y al soporte de la compra de "vehículos limpios", mediante medidas a nivel nacional, regional y local, apoyadas por la Unión. No menos importante es la promoción de renovables, más concretamente en lo referente a la relación de las mismas con la I+D+i; así como a la creación de industria y empleo relacionada con aquellas tecnologías renovables en las que se desea mantener un liderazgo, que se reconoce amenazado por otras regiones o países.

Esta Unión Energética no es fácil de entender sin la influencia de las políticas en Francia y Alemania con su "filosofía" de transiciones energéticas. En Francia con la Ley n ${ }^{\circ}$ 2015-992 de 17 de agosto de 2015 relativa a la transición energética para un crecimiento verde, y en Alemania con el Energiewende; cuya influencia sobre la energía, la competitividad y la reindustrialización constituyen, per se, un elemento que merece una consideración aparte.

\subsection{La transición energética en Alemania: Energiewende}

La transición energética, entendiendo como tal los cambios fundamentales en las estructuras o en la composición de las energías primarias y finales, es un proceso de larga duración y continuado en el tiempo. Las transiciones energéticas que se han dado en el pasado son el resultado de un conjunto de factores, entre ellos los económicos o tecnológicos, que no logran "eliminar" aquellas energías que, al inicio del proceso, tienen un peso porcentual más relevante en el mix energético ${ }^{23}$.

En esas transiciones energéticas no siempre han sido visibles objetivos políticos manifiestos. En la actualidad, sin embargo, y en el caso de Alemania en particular, los objetivos y la determinación política son fundamentales. Si bien la

\footnotetext{
${ }^{23}$ Smil, V., Energy Transitions: History, Requirements, Prospects, 2010.
} 
estructura energética del país ha ido cambiando desde el fin de la segunda Guerra Mundial, puede señalarse el año 1990 como un hito del actual proceso ${ }^{24}$, cuando el Gobierno alemán aprobó la primera Ley de fomento de las energías renovables no convencionales. Posteriormente, en 2000, se aprobó la Ley de Energías Renovables, y en la primavera de 2011 el Energiewende, articulado en base a lo anterior y al cierre de las centrales nucleares (12,5 GW entre 2015-2022).

Un elemento clave del Energiewende, es el incremento del porcentaje de renovables para llegar en generación eléctrica, a un 38\% en 2020, a un 50\% en 2030, y a un $80 \%$ en 2050 y, en términos de energía final, lograr el 18\% en 2020, 30\% en 2030 y $60 \%$ en 2050. También son clave los objetivos de eficiencia energética: reducción de un $20 \%$ del consumo de energía primaria en 2020 y un $80 \%$ en 2050; reducción de un 10\% en el consumo de energía eléctrica en 2020 y 25\% en 2050 (en comparación con niveles de 2008) y una reducción del 20\% de la demanda para el consumo de calefacción. En este ámbito, el país invierte muy fuertemente en la industria relacionada con la eficiencia energética, y se espera que este mercado se duplique en el año 2020, en relación con 2005, cuando ascendió a $450.000 \mathrm{M}^{25}$.

Para entender el Energiewende hay que situarlo en el contexto de una búsqueda sistemática de generación descentralizada, con propiedad de particulares y cooperativas; y no considerarlo como un proceso aislado. Ha de verse coordinado con la industria de suministro de bienes, equipos y servicios, en los ámbitos de eficiencia energética, solar y eólica, utilizando la base doméstica, como plataforma para las exportaciones. Por otro lado, previsiblemente que se vaya a un sistema con más costes fijos y menos variables, donde se gestionarán sistemas energéticos mucho más complejos, surgiendo nuevos modelos de negocio.

Es interesante señalar el efecto de arrastre de la energía sobre la industria. A modo de ejemplo, en 2014, cuatro empresas alemanas de energía eólica (ENERCON, Senvion, Nordex y Siemens) tenían el 68,9\% del mercado alemán; además Siemens tenía casi el 10\% del mercado mundial de aerogeneradores y ENER$\mathrm{CON}$ el 7,8\%, mientras que Gamesa se situaba en el octavo puesto, habiendo pasado del $11,5 \%$ en 2003 , al $8 \%$ en 2011 y al $4,7 \%$ actual.

La energía solar fotovoltaica y la eólica son las principales tecnologías de generación renovable sobre las que Alemania está basando su transición energética y su plataforma para las exportaciones. Así, la industria fotovoltaica alemana exportó el 60\% de su producción en 2012, frente al 14\% en 2004. En el caso de la energía eólica alemana, actualmente se estima que las exportaciones suponen entre un 65 y un $70 \%$ de las ventas.

\footnotetext{
${ }^{24}$ Los orígenes de Energiewende pueden identificarse con los movimientos antinucleares de 1968 y la búsqueda de alternativas energéticas más allá de los combustibles fósiles y la energía nuclear.

${ }^{25}$ Berger, R., Market potential in energy efficiency in Southeast Asia, 2011.
} 


\section{Eloy Álvarez Pelegry}

El Energiewende supone también la construcción de 5.000 km de redes eléctricas para 2022, para transportar la energía del norte, en gran parte eólica off-shore, con un presupuesto de $3.500 \mathrm{M€}^{26}$, así como reforzar otros $5.400 \mathrm{~km}$ de líneas ${ }^{27}$.

Además, cabe destacar que los consumidores alemanes han soportado fuertes incrementos en los precios de la electricidad ${ }^{28}$, si bien han recaído principalmente sobre los consumidores domésticos, puesto que carecen de las exenciones que en Alemania tiene la industria (ver apartado 3.1).

\subsection{Los "Prosumidores": protagonismo del consumidor individual final y la energía distribuida}

En Europa, probablemente influido por la política y la industria de bienes de equipo relacionados con la energía, el consumidor individual final puede cobrar protagonismo, en base al despliegue de los contadores inteligentes y a la tendencia hacia la generación a pequeña escala, bien para autoconsumo o bien para integrar las energías renovables de relativamente poca potencia. Estrechamente relacionado con lo anterior surge el concepto de Smart Energy, que agrupa términos como digitalización, smart home, customer engagement, big data, demand response y otros.

El sector eléctrico está sufriendo numerosos cambios, como consecuencia de las nuevas políticas energéticas, la penetración de fuentes renovables y la generación distribuida. Además, el consumidor es más activo y exigente. Estas tendencias llevan a una revisión del diseño y de las funciones de las nuevas redes de distribución ${ }^{29}$ y plantea cómo las existentes deben adaptarse para convivir con los nuevos requerimientos. Las tecnologías y la regulación juegan aquí un papel determinante. En este entorno cambiante, diferentes agentes están posicionándose en el sector eléctrico con nuevos modelos de negocio. Por todo lo anterior, las utilities eléctricas deben enfrentarse a nuevos retos y oportunidades ${ }^{30}$.

El énfasis de los consumidores finales también debe de verse como un desplazamiento a la búsqueda de un menor consumo de energía en favor de la eficiencia,

\footnotetext{
${ }^{26}$ Sánchez, R., "La gran autopista eléctrica de Alemania”, El Mundo, 13/02/2014.

${ }^{27}$ Von Hirschhausen, C., "The German "Energiewende". An introduction". Economics of Energy $\mathcal{E}^{2}$ Environmental Policy, 2014. y Fundación Focus-Abengoa. Foro transición energética y cambio climático - la Energiewende alemana, 2015. Retrieved from http://www.transicionenergeticaycc.org/web/es/iniciativaen-otros-paises/la-energiewende-alemana/

${ }^{28}$ Eurostat, Energy price statistics, 2015. Retrieved from http://ec.europa.eu/eurostat/statistics-explained/index.php/Energy_price_statistics

${ }^{29}$ Para más información ver Castro, U. y otros, El Futuro de la Redes Eléctricas, Cuadernos de OrkestraCátedra de Energía, 2013. Y Álvarez, E.; Castro, U., Generación Distribuida y Autoconsumo. Análisis regulatorio, Cuadernos de Orkestra-Cátedra de Energía, 2014. deusto.es

${ }^{30}$ Abella, A. y otros, "Smart Energy: nuevas aplicaciones y modelos de negocio. 2015. www.orkestra.
} 
bien sea en los edificios, en los hogares, o en la participación del consumidor final desde el lado de la demanda. Esta tendencia no es nueva, pero progresivamente cobrará mayor importancia en varios países europeos.

\subsection{El transporte. Una transición en ciernes}

El transporte es un tema que tiene, como la energía, numerosas y variadas facetas que van desde la aplicación de los conceptos y objetivos de movilidad sostenible, al estudio de los diversos modos de transporte (carretera, ferrocarril, aéreo y marítimo), a las infraestructuras y su gestión.

A nivel europeo, el 60\% de las emisiones de $\mathrm{CO}_{2}$ del transporte, son debidas al transporte de pasajeros y el $40 \%$ restante al de mercancías. Del primero, un $17 \%$ corresponde a trayectos urbanos y un 33\% a los interurbanos ${ }^{31}$. Entre los objetivos que la Comisión Europea ha establecido se encuentra el desarrollo y la utilización de nuevos combustibles y sistemas de propulsión sostenibles; así como, la reducción a la mitad del uso de combustibles de "propulsión convencional" en el transporte urbano en 2030, y su eliminación progresiva de las ciudades para el año 2050. Existen también otros objetivos para los sectores aéreo y marítimo, así como los referentes a la optimización del rendimiento de las cadenas logísticas multimodales, incrementando el uso de modos más eficientes de transporte desde el punto de vista energético.

Un aspecto de interés es la estrategia tecnológica. Reconociendo que no se puede dejar de depender del petróleo de una manera drástica, las innovaciones tecnológicas pueden lograr una transición más rápida y económica. En este caso, la eficiencia de los vehículos mediante nuevos motores, materiales y diseños; el recurso a una energía más limpia mediante nuevos combustibles y nuevos sistemas de propulsión; así como una mejor utilización de las redes y unas operaciones más seguras, mediante los sistemas de información y comunicación; son, todos ellos, elementos a considerar en una estrategia de movilidad sostenible.

Puede decirse que existen continuos avances en las tecnologías del motor de combustión interna (ICE), en los vehículos eléctricos híbridos y de baterías, y también en los vehículos a gas, sea gas natural comprimido (GNC) o licuado (GNL). Así en los vehículos de propulsión convencional el rendimiento ha mejorado situándose las emisiones de $\mathrm{CO}_{2}$ por $\mathrm{km}$ en $130 \mathrm{~g} / \mathrm{km}^{32}$.

${ }^{31}$ Comisión Europea, White paper. Roadmap to a Single European Transport Area - Towards a competitive and resource efficient transport system. Libro Blanco, 2011.

http://eurlex.europa.eu/search.html?DB_AUTHOR=commission\& textScope0=ti\&DTA=2011\& qid=1433420196688\&FM_CODED=WHITEPA\&DTS_DOM=EU_LAW\& typeOfActStatus=OTH ER\& type $=$ advanced $\&$ lang $=$ en $\&$ and $T$ ext $0=$ white $\% 20$ paper\&SUBDOM_INIT $=$ PRE_ACTS\&DTS_ SUBDOM=PRE_ACTS.

${ }^{32}$ European Environment Agency, Monitoring $\mathrm{CO}_{2}$ emissions from passenger cars and vans in 2013, 2014. Disponible en: http://www.eea.europa.eu/publications/monitoring-co2-emissions-from-passenger 


\section{Eloy Álvarez Pelegry}

En la comparación entre vehículos ICE y eléctricos (EV o HEV) ${ }^{33}$, las diferencias de precio y de autonomía siguen siendo una barrera para éstos últimos, junto con la necesidad de nuevas infraestructuras. Ahora bien, ha habido una continua disminución del precio (en términos de $€_{2010} / \mathrm{kW}$ ) y mejora de rendimiento desde 1997, cuando se lanzó al mercado japonés el primer modelo hibrido eléctrico ${ }^{34}$. En base a extrapolaciones sobre la curva de aprendizaje, se requiere un gran volumen de ventas para lograr un precio similar al de los vehículos ICE en los EV o HEV.

La importancia de la electrificación del transporte, en el contexto de la transición energética, es muy clara en Francia. Así las leyes "Grenelle 1, Grenelle 2, y Grenelle 3", que se refieren a la edificación y el urbanismo, incluyen también el transporte y la energía. La segunda fija como objetivos el desarrollo de los vehículos eléctricos e híbridos recargables y la creación y el mantenimiento de las infraestructuras de recarga necesarias en las colectividades locales, las viviendas y los lugares de trabajo. Francia, con más de 25.000 vehículos 100\% eléctricos a finales del año 2013, aspira a tener entre 450.000 y 800.000 vehículos EV y HEV en el horizonte de 2020, cifra revisada a la baja respecto a la anterior.

También el gas busca oportunidades para crecer en el transporte. En el mundo existen más de 16 millones de vehículos a gas, y Europa aboga por un mayor uso de gas en el transporte (Plan de Acción Red TENT-T), que promueve los "corredores azules" con puntos de carga para GNC y GNL. Las ventajas que el gas natural tiene como combustible sustitutivo, en particular del gasoil en zonas urbanas con elevada contaminación, hace que la penetración de esta energía, sea un elemento también a considerar. De ahí el énfasis en incrementar el número de vehículos a gas (100.000 en Alemania, 4.600 en España ${ }^{35}$ ) y el número de estaciones de servicio (Europa 2.400 y España 48).

\section{TRES RETOS PARA LA ENERGÍA EN ESPAÑA}

En el contexto global y europeo, al que se ha hecho referencia en el apartado anterior, pueden identificarse numerosos retos. Como ya he comentado, en $\mathrm{mi}$ opinión, son tres los retos fundamentales.

\footnotetext{
${ }^{33} \mathrm{EV}$ = vehículos eléctricos; $\mathrm{HEV}$ = vehículos híbridos-eléctricos.

${ }^{34}$ Weiss y otros, "On the electrification of road transport. Learning rates and prices forecasts for hybrid-electric and battery electric vehicles". Energy Policy, September 2012.

${ }^{35}$ Europa Press, El número de vehículos a gas se duplica en cinco años, 2015, disponible en: http://www.europapress.es/motor/coches-00640/noticia-numero-vehiculos-gas-duplica-cinco-anos-20150605112952. html
} 
El primero es el de la competitividad. Dicho reto, ha cobrado relevancia, en particular tras la persistencia de precios de la energía elevados, durante la segunda parte de la década pasada. Hoy, se advierte un cierto relajamiento, como consecuencia de la caída del precio del crudo y del gas.

El segundo reto, es uno de los "habituales", y es condición sine qua non, para la economía y la sociedad, a saber el de la seguridad de suministro. En él se pueden incluir aspectos de carácter más general, como la salud y el medio ambiente ${ }^{36}$. La seguridad de suministro, es un "viejo conocido", y cuenta con numerosos instrumentos para su consecución. Ésta requiere estar siempre vigilante y promover la implementación de nuevos enfoques, algunos evidentes, como la identificación y el eventual desarrollo de los recursos domésticos, a los que las políticas energéticas e industriales no prestan la debida atención.

El crecimiento no es un reto que habitualmente se explicite en la industria energética. Sin embargo, la crisis económica, la incertidumbre en las perspectivas de crecimiento, los objetivos de eficiencia energética y los desarrollos y acontecimientos en los últimos años, hacen que sea un reto de primera magnitud. Relacionado, estrechamente, con la competitividad y el crecimiento, está la reindustrialización ${ }^{37}$. Aquí el efecto de la energía y su crecimiento es doble. Por un lado, porque los inputs energéticos afectan a la competitividad industrial, y por otro, porque en la operación y mantenimiento de las instalaciones y, sobre todo, en las inversiones en nuevas instalaciones, la industria es un suministrador y un acompañante clave.

\subsection{Energía para la competitividad industrial}

Al referirse a la energía para la competitividad empresarial, se puede pensar, en primer lugar, en los precios de la energía y en el porcentaje que ésta supone respecto a los costes de explotación. Ciertamente este es el ratio más evidente. No obstante, no es el único parámetro a considerar.

En lo que respecta a los factores que contribuyen a la competitividad, la productividad es un parámetro determinante ${ }^{38}$. Relacionado con lo anterior, la innovación y el tamaño empresarial son elementos clave y, en algún caso, también determinantes. Para medir la productividad, entre otros ratios, se considera el de Valor Añadido Bruto (VAB). La competitividad de una economía puede, entonces, definirse como su capacidad para mantener o aumentar, de manera sostenida,

\footnotetext{
${ }^{36}$ Estos aspectos, que son factores de contexto y/o retos importantes, no se tratan en este trabajo.

${ }^{37}$ Sobre el mismo se ha llamado ya la atención en Álvarez, E. y Larrea, M. Energy policy: European challenges, Spanish answers. Policy paper 106, March 2014. Notre Europe. Jacques Delors Institute. http:// www.institutdelors.eu/media/energyeuandspain-alvarez-larrea-ne-jdi-mar14.pdf?pdf $=$ ok

${ }^{38}$ Aranguren, M. J. y otros, Estrategias para la construcción de ventajas competitivas regionales. Orkestra, Marcial Pons, 2012.
} 


\section{Eloy Álvarez Pelegry}

la presencia de las empresas en los mercados mundiales, incluyendo el propio mercado interior ${ }^{39}$.

Si bien el coste de la energía sobre los costes de explotación, no es el único parámetro a considerar, conviene comenzar identificando los sectores industriales en los que los gastos energéticos (electricidad, gas, y otros combustibles) suponen un mayor peso sobre los costes de explotación. En base a datos del periodo 20102012 se identifican: la metalurgia (17\%), los minerales no metálicos (vidrio y cemento) $(12 \%)$, el papel (10\%), la madera (8\%), la química (7\%), y el caucho y plástico $(6 \%)^{40}$.

Si se considera el desglose entre electricidad, gas y otros combustibles, en el período 2010-2012 puede advertirse el importante peso de la electricidad y, a su vez, la relevancia del consumo de gas en sectores como el vidrio o la química. Si bien todos los datos son significativos, lo son más los ratios de costes energéticos sobre el VAB del que ya se ha indicado su relevancia para la productividad. Los porcentajes se elevan a $42 \%$ para metalurgia, $30 \%$ vidrio y cemento, $18 \%$ papel, $16 \%$ madera y caucho, $17 \%$ química y $11 \%$ caucho y plástico.

Además de ser grandes consumidores, desde el año 2005, los precios de la energía, tanto gas como electricidad han aumentado ${ }^{41} \mathrm{y}$, en general, la evolución de los precios de los productos y del volumen de ventas (a pesar del incremento de las exportaciones) no han podido compensar el incremento de precios de la energía.

Reconociendo que la competitividad no depende únicamente de los costes energéticos, éstos tienen un peso decisivo, especialmente en los sectores más intensivos en energía y en aquéllos que compiten con instalaciones, con tamaño y características similares, pero que tienen costes energéticos menores. De igual manera, los costes de la energía son un parámetro de gran sensibilidad.

A modo de ejemplo, en Alemania, existe, una exención en el precio de la electricidad para la industria con un consumo de electricidad superior a $1 \mathrm{GWh} /$ año y un gasto en electricidad que represente, al menos el 14\% del VAB. Exención que tiene valores crecientes (i.e. de $10 \mathrm{GWh} /$ año a $100 \mathrm{GWh} /$ año, solo paga el $1 \%$ de la prima de renovables-1\%EEG-Umlage-). Entre los sectores a los que se aplica lo anterior, se encuentran la siderurgia y metalurgia, químicas y farmacéu-

\footnotetext{
${ }^{39}$ Servicio de Estudios del Banco de España, El Análisis de la Economía Española. Ed. Alianza Editorial, Madrid, 2005.

${ }^{40}$ Larrea, M. y otros, Precios de energía y competitividad industrial, Documento interno de trabajoCátedra de Energía.

${ }^{41}$ En el período 2000-2013 el aumento del precio del gas en Japón fue del 243\%, 271\% en Alemania, 292\% en España y - 12\% en EE. UU. En electricidad España ha experimentado, en el periodo 2007-2014, el mayor crecimiento de precio para el consumidor industrial, en el entorno del $45 \%$, frente a aumentos del 26\% en Alemania y Francia.

Las industrias citadas suponen el $70 \%$ del consumo de electricidad en la industria manufacturera en Alemania y el 27\% del consumo total de energía eléctrica en Alemania.
} 
ticas, fabricantes de vidrio y cemento, y productores de papel, cartón y plásticos ${ }^{42}$. Las industrias citadas suponen el $70 \%$ del consumo de electricidad en la industria manufacturera del país y el $27 \%$ del consumo total de energía eléctrica.

Estas exenciones han sido objeto de un estudio reciente, que compara los precios finales de electricidad para los sectores de la química, papel, aluminio, cobre y textiles, y para esas industrias, en países como Holanda, Reino Unido, Francia, Italia, Dinamarca, Canadá, EE. UU, China, Corea y Japón ${ }^{43}$. La comparación muestra que los consumidores intensivos en energía, pagan la electricidad más barata en Alemania, que en los países citados. El precio, según el estudio es de unos 50€/MWh, pero sería de unos 120€/MWh, si se le sumasen los recargos por renovables, y los impuestos. Es decir, hay una diferencia de más de 70€/MWh como consecuencia de las exenciones.

\subsubsection{Sugerencias de mejora}

Retomando el punto de los precios energéticos, el crecimiento de éstos tiene raíces muy distintas. En la electricidad, se debe fundamentalmente al incremento del coste de los peajes, a los que se les asignan el coste de las primas del anteriormente denominado régimen especial, que crecieron casi exponencialmente ${ }^{44}$, como consecuencia de un sistema de primas elevadas y un desarrollo mayor que el planificado; lo que contribuyó a un déficit de ingresos del sistema, y a la necesidad de una reforma eléctrica que se inició en $2013^{45}$.

En el año 2007, las primas al régimen especial supusieron unos 2.100 millones de euros. En 2013, superaron los 9.000 millones. Por su parte, los costes de las actividades reguladas suponían en 2007 algo más de 5.000 millones de euros, frente a los 20.000 de $2013^{46}$. Es decir, como consecuencia de una serie de medidas, que no son objeto de valoración ni de evaluación aquí, en 2010, los peajes de acceso y los impuestos supusieron el $68 \%$ de los costes del sistema. Es decir, lo que se puede denominar políticas Medioambientales, Industriales y Sociales (Políticas

\footnotetext{
${ }^{42}$ Bundesamt für Wirtschaft und Ausfuhrkontrolle (BAFA), Durch die Besondere Ausgleichsreglung in begünstigte Abnahmestellen. Herausgegeben vom Bundesamt für Wirtschaft und Ausfuhrkontrolle, Eschborn, Germany, 2014. Disponible en http://www.bafa.de/bafa/de/energie/energie_aktuell/index.html

${ }^{43}$ Fraunhofer Iso, Ecofys, Electricity Costs of Energy Intensive Industries. An international Comparison, 2015. p. 57.

${ }^{44}$ Álvarez, E., "La energía en España: Análisis y reflexiones". Estudios Empresariales (146; 2014/3),

${ }^{45}$ Díez, A. y otros, De la liberalización [Ley 54/1977], a la reforma [Ley 24/2013] del sector eléctrico español, Cuadernos Orkestra-Cátedra de Energía, 2015.

${ }^{46}$ Dentro de este concepto hay que incluir los diferentes impuestos que cargan la electricidad. En el caso del consumidor industrial el tipo impositivo en España es de 5,57 €/MWh y el IVA el 21\%. A estas cifras hay que sumar el 7\% sobre la producción de electricidad. Larrea, M.; y otros, Revisión de los impuestos energéticos en España. CEPRIE, 2014. Disponible en www.foes.de
} 


\section{Eloy Álvarez Pelegry}

MINSO $)^{47}$ encarecieron el precio de la electricidad. Teniendo en cuenta que el coste de estas políticas medioambientales, industriales y sociales está siendo soportado por los consumidores eléctricos, sería conveniente descargar a la tarifa de las mismas ${ }^{48}$ y hacer recaer su peso sobre el conjunto de ciudadanos. De esta manera, el consumidor eléctrico soportaría los costes que le son propios, es decir, los de generación, transporte y distribución.

En lo que al gas se refiere, como ya se ha comentado, puede afirmarse que existe una estrecha relación entre los precios del gas importado en España con los precios del crudo. La influencia del mercado spot ligado a las importaciones de GNL, no llegan a representar un porcentaje tan significativo, ni supone una influencia que afecte a la mayoría del mercado ${ }^{49}$. Si esto fuera así, ¿conviene promover otros mecanismos de formación de precios que sean de interés para la industria consumidora de gas que redunden en su beneficio cuando hayan de competir con la industria en Europa? A nivel de la UE si bien existe también una relación importante entre el precio del crudo y el del gas natural, ésta ha ido disminuyendo con el tiempo, pasando la formación de los precios vía indexación al crudo del 78\%, en el año 2005, al 42\% en $2013^{50}$.

Esta situación no es ajena al desarrollo de los hubs de gas o mercados organizados de gas en Europa Continental, que han ido ganando volumen, liquidez y "rotación", tanto en los hubs más importantes como el TTF o Zeebrugge, como los relativamente más recientes como GASPOOL, PEG Nord y PEG Sud, en los que se observa una tendencia a la integración de zonas de mercado.

Estos mercados facilitan plataformas y son punto de encuentro entre compradores y vendedores, que mediante productos estandarizados, bien a corto (un día; un mes) o a medio plazo, permiten adquirir gas. Además, y no menos importante, permiten conocer los precios de los diferentes productos y, por ello, facilitan señales que dan indicaciones a los compradores y vendedores sobre la situación del mercado.

Un gran volumen de gas sigue negociándose mediante contratos bilaterales (OTC), en condiciones acordadas por las partes; no siendo el precio resultante de conocimiento público. En España se realizan numerosos intercambios de gas

${ }^{47}$ Álvarez, E. y Castro, U., Generación Distribuida y Autoconsumo, Cuadernos Orkestra-Cátedra de Energía, 2015.

${ }^{48}$ Lo que se podría llevar a cabo de manera progresiva.

${ }^{49}$ En el XVII Encuentro Especializado del Sector Gasistas Español, 1 octubre 2015, se puso de manifiesto una fórmula que puede representar la relación típica ente el precio del crudo y del gas natural: precio gas $(\$ \mathrm{MMBtu})=0,8$ Precio Brent $(\$ / \mathrm{bl})+(2-4 \$ \mathrm{MMBtu})$. Una discusión sobre la relación entre precios del crudo y precios del gas puede verse en Álvarez, E. y otros. Del yacimiento al consumidor, 2000. Cie Inversiones Editoriales Dossat.

${ }^{50}$ International Gas Union, IGU, Wholeshale gas price survey. A global review of price formation mechanisms 2005-2013, 2014. 
sin precio visible ${ }^{51}$, salvo para la TUR, el gas colchón y el gas de operación. Sin embargo, no cabe duda, de que a medida que se desarrollan los hubs, los volúmenes de contratación crecen, y el número de agentes aumenta, por lo que mayor es la repercusión y la relevancia que tienen las señales de precio de estos hubs y, previsiblemente y de forma progresiva, los precios serán también utilizados como referencia para contrataciones que no se realicen por dichos mercados.

En todo caso, mientras en la Península Ibérica no se desarrolle un hub de gas, las diferencias de precios de éste, con países del entorno, no beneficiarán a la industria española, en especial, aquélla que siendo intensiva en energía tenga en el mercado europeo, y en especial en Francia, gran parte de su mercado (i.e., vidrio, cemento o papel). Así, el desarrollo de un hub de gas ibérico y el aumento de interconexiones con Francia, permitiría previsiblemente converger los precios del gas, y por ello la desventaja en los costes del gas podría desaparecer mitigando o eliminando una desventaja sino competitiva; sí comparativa.

La Ley 8/2015, de 21 de mayo, estableció las bases para el desarrollo del mercado organizado del gas en Iberia, mediante la adaptación de la sociedad MIBGAS. Por otra parte, el proyecto de Real Decreto por el que se regula el mercado organizado de gas (MOG), y el acceso de terceros (ATR) a las instalaciones del sistema de gas natural da un paso en su desarrollo pero faltan las reglas de mercado. Es posible que haya que esperar al cuarto trimestre de 2016, para que el mercado sea una realidad operativa para los consumidores industriales. Además, hay que tener en cuenta que no todos los productos van a ser objeto de negociación. Previsiblemente, se comenzará por productos estándares que van desde los intradiario, diario y mensual, a los quince años; siendo coherentes los primeros con el mercado a corto plazo (del día al mes). Además se crearán productos estándar de contratación de capacidad de almacenamiento, inyección, extracción, descarga de buques, regasificación, almacenamiento de GNL, cisternas, carga de GNL a buque, trasvase de GNL a buque y licuefacción virtual.

Una parte de estos productos tiene posiblemente más interés para las comercializadoras y para el gestor técnico del sistema, en la medida en que las reglas de balance supondrán equilibrar diariamente las entradas y salidas de gas. Posiblemente para los consumidores industriales, tengan más interés productos más allá del diario, y en particular, aquellos con un horizonte temporal anual.

Pero no cabe duda, de que el mercado organizado constituirá un avance, y si el desarrollo va acompañado de las interconexiones con Francia, que soporten flujos físicos y virtuales con volúmenes crecientes, parte de las desventajas comparativas se mitigarán o desaparecerán para aquellas industrias o productos cuya competencia directa esté en Francia y, por extensión, en Centro Europa. En todo

${ }^{51} \mathrm{CNMC}$, Informe de supervisión del mercado mayorista y aprovisionamiento de gas. Periodo de noviembre de 2014. Madrid, 2015, CNMC. 


\section{Eloy Álvarez Pelegry}

ello, no debe de olvidarse que el conocimiento de los mercados y la organización y capacidad de compra, son elementos muy importantes para que las adquisiciones se realicen de la forma más inteligente y competitiva posible.

\subsection{La seguridad de suministro y el desarrollo de recursos propios}

$\mathrm{Al}$ referirse a la seguridad de suministro de energía se entiende que los consumidores disponen de la energía final (productos petrolíferos, gas, electricidad, etc.), de forma continuada y accesible cuando se requiera.

Los procesos de transformación del crudo en productos petrolíferos (gasóleo, gasolinas, etc.), y de la generación de electricidad (a partir de gas, renovables, carbón,...), y en el caso del gas natural de la regasificación; se deben de considerar en términos de seguridad (por ejemplo, fiabilidad, disponibilidad, etc.). En el caso de productos almacenables (gas, crudo, productos petrolíferos) también hay que considerar la capacidad de almacenamiento. Además, una vez que la energía ha pasado por los procesos de transformación, se debe tener en cuenta su transporte y distribución hasta el consumidor final; lo que plantea la cuestión de la fiabilidad de los sistemas de transporte y distribución de petróleo, gas y electricidad ${ }^{52}$.

Una segunda concepción, habitual, al referirse a la seguridad de suministro, es la referente a la posibilidad de que siendo el crudo y el gas, en su práctica totalidad importados, el suministro no llegue en las cantidades necesarias o en el momento oportuno a los puntos de entrada del país importador. Aquí la diversificación y los almacenamientos, así como las instituciones responsables de la gestión de los sistemas y los almacenamientos, juegan un papel fundamental ${ }^{53}$.

En este caso, los recursos domésticos no presentan el mismo tipo de riesgos a los que están expuestas las importaciones de terceros países, entre otros a riesgos geopolíticos y, al mismo tiempo, contribuyen a mejorar la balanza comercial. Hoy en día, el precio reducido del crudo podría llevar a pensar a los agentes que temas como la producción de shale oil en EE. UU, la situación geopolítica en Irak e Irán y la mitigación de la crisis del gas en Ucrania, por ejemplo, les son ajenas, llegando a considerar que el tema no es relevante. No obstante, la experiencia muestra cómo los aspectos geopolíticos son muy relevantes y las incertidumbres permanentes en el entorno internacional, por lo que los recursos propios ofrecen más seguridad.

\footnotetext{
${ }^{52}$ No se profundiza aquí en este aspecto de la seguridad de suministro, remitiendo al lector interesado a Álvarez, E., "La fiabilidad del sistema eléctrico", Aranzadi, C., López, C. (eds) Energía, Tecnología, Economía y Regulación; Academia Europea de Ciencias y Artes, 2015, en el caso de la electricidad, y Álvarez, E., (2014) "La Energía en España: análisis y reflexiones", Estudios Empresariales (núm. 146, cuatrimestre 2014), pp. 57-74, para el gas y el petróleo.

${ }^{53}$ Álvarez, E., "La Energía en España: análisis y reflexiones", Estudios Empresariales (núm. 146, cuatrimestre 2014), pp. 57-74.
} 
Si se examina la evolución de la energía primaria en España en los últimos veinticuatro años, se observa que el petróleo ha disminuido su peso en mix pasando de representar el 49\% en el año 1990 al 42,9\% en 2014. El gas natural, por su parte, que representaba el 6\% en 1990 pasó a suponer el 20\% de la energía primaria en $2014^{54}$. Es decir, el gas natural, ha cobrado un fuerte peso en el mix, ganando los hidrocarburos ocho puntos porcentuales. No menos importante, en el caso del gas, es que su peso en el mix energético probablemente crezca debido al progresivo desarrollo del transporte y la distribución ${ }^{55}$. Además se espera, como ya se ha comentado, que aumente la penetración del gas en el transporte terrestre y marítimo junto con una mayor utilización en las centrales de ciclo combinado. En cualquier caso, los porcentajes, tanto de petróleo como de gas natural son ya muy significativos.

Por tanto, a pesar de una mayor penetración de las fuentes energéticas renovables y un efecto positivo de la eficiencia energética, no cabe duda de que los hidrocarburos seguirán teniendo una gran importancia en el mix energético. Por ello, la exploración de recursos domésticos y su eventual explotación, tienen gran sentido en términos de seguridad de suministro y mejora de la balanza comercial.

\subsection{Crecimiento y reindustrialización tras las crisis}

Ya se ha comentado, al situar en el contexto los temas energéticos, cómo el ámbito global está sujeto a crecimientos desiguales y a considerables incertidumbres, con importantes cambios demográficos. Estas desigualdades son también muy claras en la energía, siendo una de las más llamativas la evolución de la demanda de energía entre Asia (aumento del 60\% de aquí a 2040) y la OCDE (sólo un 3\% de crecimiento) ${ }^{56}$.

En España, el crecimiento económico de los últimos quince años ha mostrado una notable variabilidad, con importantes crecimientos previos a la crisis de 2008 (4,2\% en 2006) y fuertes caídas en los años siguientes, hasta 2013 inclusive, con disminuciones acusadas ( $-3,6 \%$ en 2009$)$; habiendo perdido un $7-8 \%$ del PIB, en el periodo 2008-2013.

\footnotetext{
${ }^{54}$ CORES, Consumo de productos petroliferos, 2015, disponible en: http://www.cores.es/es/estadisticas.

${ }^{55}$ Enagás, con una visión a largo plazo, tiene prevista la realización de nuevas inversiones (430 millones de euros anuales) en el período 2015-2017 que pasan por desarrollar nueva capacidad de transporte, permitiendo la producción de capacidades nominales de los puntos de entrada adecuadas a las necesidades de los diferentes tipos de consumidores. Las empresas distribuidoras por su parte, tienen previsto invertir cerca de 500 millones de euros en el corto plazo para poder dar servicio a más consumidores, lo que a su vez garantizará una mayor sostenibilidad del sistema gasista.
}

${ }^{56}$ Birol, F. y otros, World Energy Outlook, Agencia Internacional de la Energía, 2014. 


\section{Eloy Álvarez Pelegry}

\subsubsection{Caída de demanda, fuertes inversiones y baja utilización de instalaciones}

En este subapartado se examinará en primer lugar, la evolución, en el período de los diez-quince últimos años, de la demanda de electricidad, gas y productos petrolíferos. A continuación y básicamente para el mismo período se apuntan varios datos relativos a las inversiones en los subsectores energéticos. En tercer lugar, se examinarán algunos ratios y datos relativos al grado de utilización de las instalaciones e infraestructuras energéticas. El examen de los puntos anteriores permite llevar a cabo algunas reflexiones sobre el efecto tractor de la energía sobre la industria.

\section{A. CAÍDA DE LA DEMANDA}

En términos de energía, la evolución de los últimos catorce años (20002014), muestra que en 2014 se estaba por debajo de los niveles de consumo del año 2000, tanto en energía primaria (118 Mtep), como en energía final (83 Mtep).

Si se pasa revista a la evolución de la electricidad, el gas natural y los productos petrolíferos; la situación y las tendencias presentan una réplica de la situación general. Así la demanda de electricidad nacional fue de 258 TWh en $2014^{57}$, similar a la de 2005. La de gas natural está prácticamente estancada en niveles de los últimos diez años, siendo en 2014 fue de 305 TWh, nivel éste inferior al del año 2004.

En cuanto al consumo de productos petrolíferos, la situación, no es halagüeña. La caída de la actividad económica, ha incidido en la actividad industrial y, así, el Índice de Producción Industrial (IPI), ha disminuido en un 18\%, en el periodo 2000-201458, lo que ha incidido en el transporte de mercancías por carretera. De esta manera el consumo de gasóleo en el año 2014 se situaba 28 millones de toneladas, cifra similar al promedio de 2001-2002, y el de gasolina alcanzó, en 2014, el 55\% de la demanda del año 2000, como consecuencia, entre otros, del proceso de dieselización ${ }^{59}$.

Por otra parte, la participación de biocombustibles no ha logrado los objetivos previstos, quedándose en un 4,6\% del consumo total de gasolinas y gasóleos en $2014^{60}$. Tampoco lo ha hecho el vehículo eléctrico, que no ha cubierto las previsiones realizadas en algunos ámbitos, como la cifra de 250.000 vehículos de

\footnotetext{
${ }^{57}$ REE, Informe del Sistema Eléctrico Español 2014, 2015. Disponible en: http://www.ree.es/es/publicaciones/sistema-electrico-espanol/informe-anual/informe-del-sistema-electrico-

${ }^{58}$ Cálculo en base a datos de Instituto Nacional de Estadística.

${ }^{59}$ CORES, Consumo de productos petroliferos, 2015, disponible en: http://www.cores.es/es/estadisticas.

${ }^{60}$ Cálculo en base CORES, Informe estadístico anual 2014, 2015, disponible en: http://www.cores.es/ sites/default/files/archivos/publicaciones/informe-estadistico-anual-2014.pdf
} 
este tipo en 2014 (o un millón de vehículos si se incluían los híbridos eléctricos a 2014 también) del Plan del Vehículo Eléctrico ${ }^{61}$, ya que en 2013 el número de unidades no llegaba a $5.000^{62}$.

\section{B. INVERSIONES EN EL SECTOR ENERGÉTICO}

En la primera década del siglo XXI, el desarrollo de infraestructuras energéticas, ha sido muy notable ${ }^{63}$. En el ámbito de la generación eléctrica, el crecimiento puede calificarse de exponencial pasando de $55 \mathrm{GW}$ de potencia nacional en el año 2000, a 108GW en el año $2014^{64}$, donde la potencia de ciclos combinados pasó de 2,7 GW a 25,3GW en el período 2000-2014. También ha sido fortísimo el crecimiento de potencia en parques eólicos y en potencia fotovoltaica, que pasó de 1,9 GW en eólica en el año $2000^{65}$ a $23 \mathrm{GW}$ en el 2014 ${ }^{66}$, y de 0,01 GW, en fotovoltaica en el año $2000^{67}$, a multiplicar por más de cuatrocientos la potencia, llegando a 4,7 $\mathrm{GW}^{68}$ en el año 2014. En cuanto a inversiones, han supuesto $60.951 \mathrm{M} €$ entre 2000 y 2013. En el año 2013 se invirtieron 2.278M€ en construcción, ampliación y mantenimiento de las instalaciones de generación y distribución de energía eléctrica ${ }^{69}$.

${ }^{61}$ Ministerio de Industria, Turismo y Comercio, Estrategia integral para el impulso del vehículo eléctrico en España, 2010. http://www.minetur.gob.es/es-es/gabineteprensa/notasprensa/documents/estrategiainteg ralveh\%C3\%ADculoelectrico060410.pdf

${ }^{62}$ Terrasa, R., “¿Por qué no arranca el coche eléctrico en España?”, El Mundo (2013), disponible en: http://www.elmundo.es/elmundo/2013/07/16/valencia/1373980924.html

${ }^{63}$ A pesar de la importancia de la inversión, las cifras pueden ponerse en contexto con las de otros sectores de importancia estratégica, como los 3.500 millones de euros invertidos anualmente por esta en infraestructuras de AVE desde el 2000, o los 8.500 millones anuales percibidos por la red de carreteras durante la última década. (Pérez, R., “¿Gastamos en AVE más de lo necesario?”, El País, junio 2015, disponible en: http://economia.elpais.com/economia/2015/06/05/actualidad/1433502101_210450.html y RACC, "El mapa vial español en los últimos 20 años: contrastes de una política de inversiones osada", Tribunas de la movilidad RACC (marzo 2012), disponible en: http://tribunas.racc.es/mapa_vial_espanyol_en_los_ultimos_20_anyos).

${ }^{64}$ Díez, A. y otros, De la liberalización [Ley 54/1977], a la reforma [Ley 24/2013] del sector eléctrico español, Cuadernos de Orkestra-Cátedra de Energía, 2015.

${ }^{65}$ REE, Informe del Sistema Eléctrico Español 2000, 2001. Disponible en: http://www.ree.es/es/publicaciones/2013/10/informe-del-sistema-electrico-espanol-2000

${ }^{66}$ REE, Informe del Sistema Eléctrico Español 2014, 2015. Disponible en: http://www.ree.es/es/publicaciones/sistema-electrico-espanol/informe-anual/informe-del-sistema-electrico-espanol-2014

${ }^{67}$ Little, A., El papel de la generación fotovoltaica en España, informe para Asociación de Productores de Energías Renovables, 2007. Disponbible en: http://www.appa.es/descargas/prensa/ADL.\%20El\%20papel_fotovoltaica\%20en\%20Espana.pdf

${ }^{68}$ REE, Informe del Sistema Eléctrico Español 2014, 2015. Disponible en: http://www.ree.es/es/publicaciones/sistema-electrico-espanol/informe-anual/informe-del-sistema-electrico-espanol-2014

${ }^{69}$ UNESA, Contribución de las Compañías que integran UNESA al Desarrollo de la Sociedad Española.2013. 


\section{Eloy Álvarez Pelegry}

Respecto a la red de transporte eléctrica, se puede hablar de inversiones del orden de 3.413M€, sólo desde el año 2010, que como cifra indicativa, supone más de 680 millones anuales ${ }^{70}$. A futuro, por ejemplo, el proyecto de interconexión entre Francia y España por el Golfo de Vizcaya supondría una inversión aproximada de 1.750 millones de euros ${ }^{71}$.

En el ámbito del gas, desde el año 2000 se han construido cuatro plantas de regasificación. Además, varias de estas plantas se han ampliado con tanques de almacenamiento. Teniendo en también cuenta las inversiones en gasoductos, se ha invertido del orden de 15.000 millones de euros entre los años 2000 y 2014, lo que ha supuesto 1.000 millones de euros anuales ${ }^{72}$. La inversión en la red gasista se ha traducido en alcanzar los 81.806 kilómetros de redes de transporte y distribución en 2014, frente a los 37.000 disponibles en el año 200073; es decir, se ha doblado la capacidad de transporte en casi quince años.

Por su parte, la capacidad actual de las refinerías está relacionada con un importante plan de inversiones planificado durante los primeros años de la década del año 2000, que se ha mantenido en el período 2008-2013 para alcanzar los 6.500 millones de euros ${ }^{74}$, y cuyo objetivo ha sido mejorar y sustituir equipos para incrementar el rendimiento, adecuarse mejor al mercado o extender la vida de las instalaciones.

Estas cifras ponen de relieve la importancia de las inversiones en el conjunto del sector energético; que debieran haber beneficiado al desarrollo de la industria nacional, tal y como se plantea en Alemania con el Energiewende.

\section{c. UTILIZACIÓN}

Las instalaciones de generación muestran un panorama de baja utilización. De las 8.760 horas anuales, los generadores eólicos y fotovoltaicos que tienen limitaciones "técnicas", han funcionado durante el año 20142.218 y 1.755 hepc (horas equivalentes a plena carga), con una producción intermitente, que necesita del apoyo de la generación convencional, fundamentalmente de gas. Únicamente el carbón y las centrales nucleares presentan un número de horas de funcionamiento que se puede considerar razonable, 4.048 en el carbón y 7.294 en nuclear ${ }^{75}$.

${ }^{70} \mathrm{REE}$, Gestor de la red y transportista. Planificación y desarrollo de la red. Acceso en octubre de 2015, disponible en: http://www.ree.es/es/actividades/gestor-de-la-red-y-transportista/planificacion-y-desarrollo-de-la-red

${ }^{71}$ Prieto, J., Interconexiones con Francia. XIV Jornadas Internacionales de Equipos Eléctricos. 2015.

${ }^{72}$ SEDIGAS, Informe anual 2014, 2015. Disponible en: http://www.sedigas.es/pagina.php? $\mathrm{p}=11$

${ }^{73}$ SEDIGAS, Informe anual 2014, 2015. Disponible en: http://www.sedigas.es/pagina.php?p=11

${ }^{74}$ Asociación Española de Operadores de Productos Petrolíferos, Memoria 2014, 2015. Disponible en: http://www.aop.es/memoria/2014/AOP\%20WEB.pdf

${ }^{75}$ Cálculos en base a REE, Informe del Sistema Eléctrico Español 2014, 2015. Disponible en: http:// www.ree.es/es/publicaciones/sistema-electrico-espanol/informe-anual/informe-del-sistema-electricoespanol-2014 
Los ciclos combinados han funcionado progresivamente menos desde el año 2008, situándose en 953 hepc en 2014, es decir una utilización del 11\%. Esta situación ha llevado a la CNMV, a analizar la cobertura estimada de los costes fijos de operación y mantenimiento de los ciclos combinados en 2014, y ha concluido que unos dieciséis ciclos combinados no habrían conseguido cubrirlos, por lo que sus propietarios podrían considerar su hibernación temporal, y/o cierre o desmantelamiento.

En el sector del gas, un parámetro que se puede emplear es el ratio de utilización de las plantas de regasificación, que ha sido del 31\% en el período 20102014. Un caso significativo es el de la planta de El Musel, que entró en hibernación de manera inmediata tras su finalización. Su construcción se planificó en un contexto de previsiones alcistas de la demanda de gas y de la necesidad de seguridad de suministro. Estas previsiones venían acompañadas por el aumento de la demanda de gas, que se esperaba, para los nuevos ciclos combinados ${ }^{76}$.

En la situación del gas natural, a la caída en el consumo por la crisis, que ha puesto cierto "techo" temporal al crecimiento del sector, se puede añadir la introducción de las energías renovables, no considerada en las previsiones, al menos en el volumen y con la velocidad con la que ha ocurrido lo que ha afectado al funcionamiento de los ciclos combinados ${ }^{77}$.

Más favorable es la situación en el refino, donde el grado de utilización de la capacidad, en 2014, fue del $82,6 \%$; 7,3 puntos porcentuales superior a la registrada en 2013. La industria española de refino procesó en sus diez plantas un total de $61,4 \mathrm{Mt}$ de crudo y materias primas; produjo 60,8 Mt de las que los gases representaron el $45,1 \%$. En el conjunto del comercio exterior, las importaciones de productos petrolíferos ascendieron a $16,6 \mathrm{Mt}$, mientras que las exportaciones alcanzaron la cifra de 19,4 Mt, impulsadas por el gasóleo ${ }^{78}$.

Así pues, en general la situación de los últimos años, en particular de 2008 a 2014, no ha sido halagüeña, desde el punto de vista de crecimiento, y ha supuesto un retroceso en la demanda. La "base" de partida, si se imagina un nuevo periodo a partir de ahora, se podría situar en niveles de hace diez o doce años. En decir, en términos de demanda en electricidad, gas natural o gasóleos estamos como en los años 2002-2005.

\footnotetext{
76 "En su momento se llegó a decir que prácticamente cada ciclo combinado debía llevar aparejada la construcción de un tanque de GNL". Llardén, A., "Los diez últimos años del sector energético en España: la visión del operador del sistema", Cuadernos de Energía ( ${ }^{\circ} 41$, diciembre 2013), Club Español de la Energía.. Disponible en: http://www.enerclub.es/es/frontNotebookAction.do; jsessionid=1F8 71CCB311D0A55C7DDE01BCC4E308B?action=viewCategory\& idCategoryToShow $=40 \&$ publication $\mathrm{ID}=1000100919$

${ }^{77}$ Llardén, A., "Los diez últimos años del sector energético en España: la visión del operador del sistema", Cuadernos de Energía (nº41, diciembre 2013), Club Español de la Energía. Disponible en: http:// www.enerclub.es/es/frontNotebookAction.do; jsessionid=1F871CCB311D0A55C7DDE01BCC4E308B ?action $=$ viewCategory\& idCategory ToShow $=40 \&$ publicationID $=1000100919$

${ }^{78}$ Revista Oil\&gas. Julio-agosto 2015.
} 


\section{Eloy Álvarez Pelegry}

\section{Mirando Al FUturo}

A futuro, varios parámetros resultan clave a nivel nacional, la evolución de la población, la de la economía, los precios relativos de las energías finales y la eficacia de las medidas de eficiencia energética. Respecto a la población, no se espera que aumente en los próximos años. Al contrario, se estima una disminución de 46,7 millones habitantes en 2013, a 44,9 en $2020^{79}$.

En cuanto al crecimiento de la economía, las estimaciones son dispares y los valores se sitúan en una horquilla del $2 \%$ al $3 \%{ }^{80}$. Además, no parece que, en el próximo quinquenio, se produzca un cambio sustancial de la estructura económica final, pero sí una sistemática caída de la intensidad de la energía final en el PIB. La electricidad y el gas mantendrán cierta estabilidad, con caídas ligeras de entre un $0,5 \%$ y un $0,8 \%$ respectivamente, hasta 2020 ; mientras las renovables aumentarían en intensidad sobre el PIB $(2,1 \%)$ y los productos petrolíferos caerían un $3 \%{ }^{81}$.

En generación eléctrica, groso modo, podría afirmarse, y a riesgo de ser poco preciso $^{82}$, que se las previsiones estiman una disminución potencial, de unos 6 GW de ciclos combinados, y un crecimiento de 4-6 GW de renovables (fundamentalmente eólica, 4,8 GW) ${ }^{83}$, con una tasa de cobertura elevada del 1,24-1,10, con una punta de potencia a nivel peninsular de entre $44 \mathrm{GW}$ y $49 \mathrm{GW}$, según distintos autores ${ }^{84}$.

Así pues, existe un potencial doméstico en renovables para cumplir con los objetivos comunitarios 2020, y a un horizonte de mayor plazo, en 2030, es previsible la necesidad de renovables, pero no sólo de generación eléctrica. Para el año 2020 las cifras de orden de la potencia necesaria, permiten afirmar que es un mercado reducido para el tamaño de empresas globales ${ }^{85}$, tema que se tratará más adelante.

${ }^{79}$ MINETUR, Propuesta de Desarrollo de la Red de Transporte de Electricidad 2012-2020.

${ }^{80} \mathrm{CNMC}$, Informe sobre la propuesta de planificación de la red de transporte de energía eléctrica 2015 2020, 2015. Disponible en: http://cnmc.es/Portals///Ficheros/Energia/Informes/20150618_Informe\%20 propuesta\%20planificacion\%202015-2020.pdf

${ }^{81}$ MINETUR, Propuesta de Desarrollo de la Red de Transporte de Electricidad 2012-2020.

${ }^{82}$ Existen otras consideraciones que llevan a que esta comparación deba precisarse. Por ejemplo, la naturaleza intermitente de algunas tecnologías.

${ }^{83}$ La AEE estima recogiendo cifras del MINETUR 4700 MW en una inversión de 6000 M€ (lo que supone 1.28 M€/MW). Fuente AEE Eólica '15.

${ }^{84} \mathrm{CNMC}$, Informe sobre la propuesta de planificación de la red de transporte de energía eléctrica 2015 2020, 2015. Disponible en: http://cnmc.es/Portals///Ficheros/Energia/Informes/20150618_Informe\%20 propuesta\%20planificacion $\% 202015-2020$.pdf

${ }^{85}$ A modo de ejemplo en el caso eólico, Gamesa en el año 2014 instaló 2.623 MW (27 MW en España). En 2008 instaló en España, aproximadamente 1.000 MW. Esto permite ilustrar cómo el mercado doméstico no puede ser la única base de reindustrialización, a partir de la energía. (Datos de los informes anuales de Gamesa 2008 y 2014). 


\subsubsection{La energía como elemento tractor}

El peso del sector energético en la economía es importante. En 2013 contribuyó en un 3,26\% al VAB y en un 2,98\% al PIB. A nivel industrial, supuso el $18,53 \%$ de la actividad. Si a estas cifras se le añaden las relativas a la actividad económica directa, indirecta e inducida de los sectores eléctricos, gas y petróleo su aportación a la economía es todavía más significativa.

Para examinar el peso económico de la actividad y su contribución directa, indirecta, e inducida, es habitual utilizar la metodología input-output. Este ejercicio se ha llevado a cabo para el sector eléctrico, para las empresas de UNESA ${ }^{86}$, que suponen aproximadamente el 75\% de la generación y el 95\% de la distribución. La contribución directa de las empresas agrupadas en UNESA se calculó en 8.636M€ (0,85\% del PIB); la indirecta consecuencia del incremento de la actividad empresarial de los proveedores directos y de los suministradores de éstos en 4.654M€ (0,45\% del PIB) y la inducida, generada por los proveedores al resto de los sectores en 7.899M€ (0,8 PIB).

En el caso particular de la industria nuclear en España, ésta aportó $2.781 \mathrm{mi}$ llones de euros al PIB español en el año 2013 nacional, con una contribución directa de 1.967 millones de euros $(0,19 \%$ del PIB); con una cadena de valor desde el aprovisionamiento, fabricación y distribución de combustible, la generación eléctrica, la gestión de residuos y el desmantelamiento de centrales, donde existe una industria auxiliar de suministro de bienes de equipo, sistemas, ingenierías, servicios e $\mathrm{I}+\mathrm{D}$.

Las cifras anteriores, que deberían completarse con las de otros sectores energéticos, que aquí no se abordan, ponen de relieve el impacto o efecto tractor de las instalaciones existentes sobre otros sectores económicos, incluida la industria.

Ya se ha comentado cómo el mercado doméstico potencial, teniendo en cuenta las inversiones de la pasada década, muestra la dificultad para crecer con fuerza con nuevas inversiones (green field). Ahora bien, en los próximos años, la mayor utilización de la capacidad instalada y la posible extensión de vida de las centrales nucleares, de carbón y eólicas ${ }^{88}$ con las mejoras y modificaciones pertinentes, pondrán de manifiesto de una forma más clara el efecto tractor de la energía sobre la industria.

En el examen de los factores de contexto que se han analizado, se ha señalado que muy probablemente surjan otras oportunidades de crecimiento y de nuevos modelos de negocio, que no sean tan evidentes como los relativos al efecto tractor de las instalaciones e infraestructuras existentes. En este sentido, pueden indicarse, al menos, tres posibilidades potenciales.

\footnotetext{
la.2013.

${ }^{86}$ UNESA, Contribución de las Compañías que integran UNESA al Desarrollo de la Sociedad Españo-

${ }^{87}$ PWC, Impacto socioeconómico de la industria nuclear en España, estudio para Foro Nuclear, 2015.

${ }^{88}$ En eólica, un número creciente de aerogeneradores tiene más de 15 años de vida.
} 


\section{Eloy Álvarez Pelegry}

En el contexto inicial, ha señalado el tema del "prosumidor", la generación distribuida y las redes inteligentes como un gran tema en el contexto de una transición energética. Esto supone una posibilidad real, cuyo impulso, no puede venir sino porque la retribución estimule y promueva la automatización y la modernización de las instalaciones ${ }^{89}$.

La segunda gran posibilidad vendrá probablemente de un conjunto de actuaciones, diversas y quizás dispersas, en materia de eficiencia energética; en mejoras de la eficiencia energética en edificios, incluyendo rehabilitaciones, y en la industria ${ }^{90}$.

El tercer gran tema es el transporte. En este ámbito, si realmente cobra impulso la transición en ciernes, las posibilidades son muy importantes para la industria, así como para las infraestructuras, tecnologías y servicios.

Finalmente una consideración que se entiende relevante. A la hora de examinar el crecimiento del sector energético, los análisis y debates habitualmente se focalizan en las renovables para la generación eléctrica. Sin embargo, no se debe olvidar el peso que el petróleo tiene en la energía primaria y sus derivados en la energía final y que, aunque su importancia relativa disminuya, seguirá siendo muy relevante. Por ello, convendría también llamar la atención sobre la cadena de valor del petróleo y del gas; identificando para ello qué elementos básicos de la cadena principal no están suficientemente cubiertos, por empresas suministradoras con sede en el territorio.

En este sentido pueden identificarse varios factores, que por su desarrollo, convendría tener en cuenta; a saber, el arrastre de las empresas de la cadena de valor principal basado en la propiedad doméstica y la capacidad de prescripción, y la capacidad de arrastre derivada de la ubicación física de las empresas y la capacidad o conveniencia de proveedores locales ${ }^{91}$. Otra consideración muy importante, a tener en cuenta, es el crecimiento basado en las expectativas de crecimiento de nuevos subsectores, como por ejemplo el shale gas.

En todos los casos, el enfoque es el de la creación de valor y de la competitividad, de tal manera que las inversiones domésticas sean una plataforma para crear una base industrial que permita competir en mercados grandes y globales.

\subsubsection{Modo de crecimiento y retos para la industria}

De los factores identificados en el contexto, y a partir del examen del crecimiento en los últimos diez-quince años, y las perspectivas que se han apuntado; parece claro que el enfoque del crecimiento lineal, que tiene su origen en la trans-

${ }^{89}$ Castro, U. y Álvarez, E., El Futuro de la Redes Eléctricas, Cuadernos Orkestra-Cátedra de Energía, 2015.

${ }_{90}^{0}$ Álvarez, E. y Mosácula, C., Energía y Edificación, Cuadernos Orkestra-Cátedra de Energía, 2013.

${ }^{91}$ Repetí, J. y Álvarez, E., La cadena de valor del Oil $\mathcal{E}$ Gas en la CAPV. Documento interno de trabajo. Cátedra de Energía-Orkestra. 
formación de la energía dirigida al consumidor final, no parece que sea el único esquema para un análisis acertado.

Ya se ha apuntado que segmentos como la eficiencia energética, el transporte y la edificación supondrán enfoques y modelos de negocio, nuevos y distintos a los habituales.

Por otra parte, y en base a los aspectos relativos al consumo final de energía y los cambios en las pautas de desarrollo que se han experimentado y conocido hasta ahora, conviene recordar que las transiciones energéticas llevan tiempo y que, salvo en las auténticas revoluciones, en un nuevo entorno, las energías existentes no desaparecen totalmente.

Debe resaltarse que, en cualquier caso, los proyectos energéticos requieren en muchos casos fuertes inversiones. A modo de ejemplo, los elevados costes específicos que presenta la energía eólica offshore a nivel mundial, con despliegues importantes, supondrá fuertes inversiones, lo que unido a desarrollos novedosos, tanto en el propio aerogenerador, como en su implantación en mar y su conexión a tierra, creará oportunidades industriales muy relevantes, en las que el tamaño y la innovación son clave.

Lo mismo puede afirmarse de las inversiones en generación convencional, sean de carbón (con instalaciones de desulfuración y desnitrificación) o nuclear; sobre las que ya se ha apuntado que a nivel global continuarán siendo mercados importantes.

Por lo anterior puede afirmarse que el hecho de que el mercado y las inversiones sean globales en ciertas tecnologías, resulta importante contar con empresas fuertes, solventes, con gran capacidad financiera, competitivas, que ganen concursos y tengan capacidad de arrastre.

Relacionado con lo anterior hay que destacar el papel que tienen la innovación y sus políticas, dada la importancia para la mejora de la productividad y de la competitividad, tanto en la disminución de costes como en la diferenciación y en la creación de nuevos productos y servicios. Estos aspectos llevarían a tratar los temas de las políticas energética, industrial y territorial para la competitividad; así como las estrategias de especialización inteligente, y la integración en las cadenas de valor global, que exceden el alcance de este artículo.

No cabe duda, que si se pretende acertar en el diseño de políticas, que propugnen el crecimiento y el bienestar, ciertos modelos o formas de enfocar los problemas, probablemente tengan que cambiar. Para ello, parece útil, repensar algunos aspectos de políticas energéticas e industriales que pueden tener relación con este tema.

\section{REFLEXIONES FINALES Y ALGUNAS SUGERENCIAS}

Llegados a este punto, me gustaría finalizar más que con unas conclusiones estructuradas de los apartados anteriores, con algunas reflexiones personales y varias sugerencias al hilo de lo expuesto en los tres retos identificados. 


\section{Eloy Álvarez Pelegry}

En el tema de competitividad es clave incidir en la necesidad de lograr unos costes de la electricidad y del gas, menores. Para ello se sugieren tres frentes de actuación. El primero sería descargar progresivamente los precios de la electricidad de aquellas partidas o componentes que no son directamente atribuibles a las actividades de generación, transporte o distribución de electricidad.

La implantación y desarrollo de mercados de gas sería el segundo frente para promover señales de precios y mecanismos de ajuste que puedan contribuir a la competitividad.

Las infraestructuras (como las interconexiones) contribuyen a la disponibilidad física y virtual del gas y de la electricidad, así como a la convergencia de los precios y mitigación de diferencias. De esta manera se reducirían las diferencias con los competidores europeos. No obstante, la posible existencia de exenciones (como en el caso alemán), introducen considerables distorsiones que deberían de corregirse.

En cuanto a la seguridad de suministro, si bien existen ya una serie de políticas, instrumentos e implementaciones, se debería de llamar la atención sobre la importancia de los recursos domésticos potenciales y a la necesidad de conocerlos mediante la exploración de los mismos.

El tercer reto es el crecimiento y aquí teniendo en cuenta la realidad de los últimos años en cuanto a la evolución de la situación económica, el retroceso en general de la demanda de energía, y las fuertes inversiones en distintos subsectores energéticos, se debería poner el énfasis en dos aspectos. Por un lado, en la necesidad de reforzar y potenciar la industria y los servicios asociados a la operación, el mantenimiento y las inversiones en modernización y extensión de la vida; al igual que en la necesidad de potenciar la cadena de valor del petróleo o el gas.

En cuanto a nuevos "segmentos" como base para el crecimiento, se sugieren los relativos al transporte y su electrificación, así como la incorporación de otros combustibles; y la eficiencia energética en la edificación y en la industria, siendo no menos importante los desarrollos de las redes eléctricas del futuro.

Es claro que, en un entorno de transición energética, las tecnologías que más crecerán en Europa serán las renovables, pero teniendo en cuenta el tamaño del mercado doméstico y sus perspectivas de crecimiento, éste no se puede abordar si no es creciendo en otros mercados y siendo competitivos en los mismos. Para ello, el tamaño es un parámetro muy relevante. La innovación por su relación con la productividad y la competitividad será también un factor igualmente necesario.

Finalmente, en el contexto de reindustrialización y teniendo en cuenta el papel y el peso que tiene la energía en la economía, sería necesario profundizar en la relación entre la energía y la industria y en revitalizar, contrastar y enriquecer los planteamientos teóricos sobre este asunto; a los que es posible que soluciones eclécticas sean más ricas y eficaces para abordar con éxito las mejores políticas. 


\section{BIBLIOGRAFÍA}

ABELLA, A. y otros, Smart Energy: nuevas aplicaciones y modelos de negocio, 2015. www.orkestra.deusto.es

AIE, World Energy Outlook 2015, 2015.

AIE, Gas Medium Term Report, 2015.

AIE, Energy Technology Perspectives. Mobility innovation to accelerate Climate Action, 2015.

ÁlVAREZ, E., "Cambio Climático; el camino a París", Expansión, (16 de mayo 2015), p. 47.

ÁlVAREZ, E., "La Fiabilidad del Sector Eléctrico", en Aranzadi, C. y López, C. (eds), Energía, Tecnología, Economía y Regulación, Academia Europea de Ciencias y Artes, Madrid, 2014.

ÁLVAREZ, E., "La energía en España: Análisis y reflexiones”, Estudios Empresariales $(146 ; 2014 / 3)$, p. 57.

ÁlVAREZ, E. y CASTRO, U., Generación Distribuida y Autoconsumo. Análisis regulatorio, Cuadernos de Orkestra-Cátedra de Energía, 2014.

ÁlVAREZ, E. y LARREA, M., Energy policy: European challenges, Spanish answers. Policy paper 106, March 2014. Notre Europe. Jacques Delors Institute. http://www.institutdelors.eu/media/energyeuandspain-alvarezlarrea-ne-jdi-mar14.pdf?pdf $=$ ok

ÁlVAREZ, E. y MOSÁCULA, C., Energía y Edificación, Cuadernos de Orkestra-Cátedra de Energía, 2013.

ÁlVAREZ, E. y otros, Energía y Tributación Medio Ambiental, Marcial Pons, 2013.

ÁlVAREZ, E. y SUÁREZ, C., El Gas No Convencional: "Shale Gas". Aspectos estratégicos, técnicos, medioambientales y regulatorios, Marcial Pons. Prevista publicación en enero de 2016.

ARANGUREN, M. J. y otros, Estrategias para la construcción de ventajas competitivas regionales. Orkestra, Marcial Pons, 2012.

ASOCIACIÓN ESPAÑOLA DE OPERADORES DE PRODUCTOS PETROLÍFEROS, Memoria 2014, 2015. Disponible en: http://www.aop.es/ memoria/2014/AOP\%20WEB.pdf

BERGER, R., Market potential in energy efficiency in Southeast Asia. 2011.

BIROL, F. y otros, World Energy Outlook, Agencia Internacional de la Energía, 2014.

BUNDESAMT FÜR WIRTSCHAFT UND AUSFUHRKONTROLLE (BAFA), Durch die Besondere Ausgleichsreglung in begünstigte Abnahmestellen. Herausgegeben vom Bundesamt für Wirtschaft und Ausfuhrkontrolle, Eschborn, 2014, Germany. Disponible en: http://www.bafa.de/bafa/de/ energie/energie_aktuell/index.html 


\section{Eloy Álvarez Pelegry}

CASTRO, U. y otros, El Futuro de la Redes Eléctricas, Cuadernos de OrkestraCátedra de Energía, 2013.

CÍRCULO DE EMPRESARIOS, Hacia una energía competitiva, sostenible y garantizada, febrero 2015.

CLEMENTE, J., "Abated Incremental Oil Demand Extends Future Supply", Forbes (abril 2015), disponible en: http://www.forbes.com/sites/judeclemente/2015/04/29/incremental-oil-demand-wont-go-unabated/

CNMC, Informe sobre la propuesta de planificación de la red de transporte de energía eléctrica 2015-2020, 2015. Disponible en: http://cnmc.es/Portals/0/ Ficheros/Energia/Informes/20150618_Informe\%20propuesta\%20planificacion\%202015-2020.pdf

COMISIÓN EUROPEA, White paper. Roadmap to a Single European Transport Area - Towards a competitive and resource efficient transport system, 2011. Disponible en http://eur-lex.europa.eu/search. html?DB_AUTHOR $=$ commission\& textScope $0=$ ti\&DTA $=2011 \& q$ $\mathrm{id}=1433420196688 \& F M \_C O D E D=$ WHITEPA\&DTS_DOM=EU_ LAW \& typeOfActStatus $=$ OTHER\& type $=$ advanced\&lang $=$ en\&an $\mathrm{dText} 0=$ white $\% 20$ paper\&SUBDOM_INIT $=$ PRE_ACTS\&DTS_ SUBDOM=PRE_ACTS

COMISIÓN EUROPEA, Road Map 2050. Disponible en https://ec.europa.eu/ energy/en/topics/energy-strategy/2050-energy-strategy

COMISIÓN EUROPEA, Paquete sobre la Unión de la Energía. Estrategia Marco para una Unión de la Energía resiliente con una política climática prospectiva. COM (2015) 80 final.

CORES, Consumo de productos petroliferos, 2015. Disponible en: http://www. cores.es/es/estadisticas.

CORES, Informe estadístico anual 2014, 2015. Disponible en: http://www. cores.es/sites/default/files/archivos/publicaciones/informe-estadisticoanual-2014.pdf

DÍAZ, A. C. y otros, De la liberalización [Ley 54/1977], a la reforma [Ley 24/2013] del sector eléctrico español, Cuadernos Orkestra-Cátedra de Energía 2015.

EUROPA PRESS, El número de vehículos a gas se duplica en cinco años, 2015. Disponible en: http://www.europapress.es/motor/coches-00640/noticianumero-vehiculos-gas-duplica-cinco-anos-20150605112952.html

EUROPEAN CLIMATE FOUNDATION, ROADMAP 2050. A practical guide to a prosperous, low-carbon Europe. Technical analysis, 2010.

EUROPEAN ENVIRONMENT AGENCY, Monitoring $\mathrm{CO}_{2}$ emissions from passenger cars and vans in 2013, 2014. Disponible en: http:// www.eea.europa.eu/publications/monitoring-co2-emissions-frompassenger 
EUROSTAT, Energy price statistics, 2015. Disponible en http://ec.europa.eu/ eurostat/statistics-explained/index.php/Energy_price_statistics

FERC, North-American LNG import/export terminals. Approved, 2015. Disponible en http://www.ferc.gov/industries/gas/indus-act/lng/lngapproved.pdf

FUNDACIÓN FOCUS-ABENGOA, Foro transición energética y cambio climático. La Energiewende, 2015. Disponible en http://www.transicionenergeticaycc.org/web/es/iniciativa-en-otros-paises/la-energiewende-alemana/

FRAUNHOFER ISO, ECOFYS, Electricity Costs of Energy Intensive Industries. An international Comparison, 2015.

GAMESA, Informe anual 2014, 2015.

GAMESA, Informe anual 2008, 2009.

INTERNATIONAL GAS UNION, IGU, Whole shale gas price survey. A global review of price formation mechanisms 2005-2013, 2014.

LARREA, M. y otros, Revisión de los impuestos energéticos en España. CEPRIE. 2014. Disponible en www.foes.de

LITTLE, A., El papel de la generación fotovoltaica en España, informe para Asociación de Productores de Energías Renovables, 2007. Disponible en: http:// www.appa.es/descargas/prensa/ADL.\%20El\%20papel_fotovoltaica\%20 en\%20Espana.pdf

LLARDÉN, A., "Los diez últimos años del sector energético en España: la visión del operador del sistema", Club Español de la Energía, Cuadernos de Energía ( $\mathrm{n}^{\circ} 41$, diciembre 2013). Disponible en: http://www.enerclub.es/es/ frontNotebookAction.do; jsessionid=1F871CCB311D0A55C7DDE01B CC4E308B?action=viewCategory $\&$ idCategory ToShow $=40 \&$ publication $\mathrm{ID}=1000100919$

MINETUR, Propuesta de Desarrollo de la Red de Transporte de Electricidad 20122020.

MINISTERIO DE INDUSTRIA, TURISMO Y COMERCIO, Estrategia integral para el impulso del vehículo eléctrico en España, 2010. http://www.minetur.gob.es/es-es/gabineteprensa/notasprensa/documents/estrategiaintegral veh\%C3\%ADculoelectrico060410.pdf

NACIONES UNIDAS, World Population Prospects. The 2015 Revision. Key findings and Advance Tables, 2015. Disponible en: http://esa.un.org/unpd/ wpp/Publications/Files/Key_Findings_WPP_2015.pdf

NACIONES UNIDAS, World Urbanization Prospects. The 2014 Revision. Highlights, 2014. Disponible en: http://esa.un.org/unpd/wup/Highlights/ WUP2014-Highlights.pdf

PARRILLI, D. y otros, Análisis de la Cadena de Valor de la Industria Eólica Vasca: Oportunidades y Ámbitos de Mejora. Orkestra-Instituto Vasco de Competitividad. Fundación Deusto, 2012. 


\section{Eloy Álvarez Pelegry}

PÉREZ, R., “¿Gastamos en AVE más de lo necesario?”, El País, junio 2015, disponible en: http://economia.elpais.com/economia/2015/06/05/actualidad/1433502101_210450.html

POPONI, D., Energy Technology Perspectives 2015: Mobilising Innovation to Accelerate Climate Action, conferencia de la Agencia Internacional de la Energía en el Ministerio Federal Austriaco de Transporte, Innovación y Tecnologías de Viena, junio 2015.

PRIETO, J., Interconexiones con Francia. XIV Jornadas Internacionales de Equipos Eléctricos, 2015.

PWC, Impacto socioeconómico de la industria nuclear en España, estudio para Foro Nuclear, 2015.

RACC, "El mapa vial español en los últimos 20 años: contrastes de una política de inversiones osada", Tribunas de la movilidad RACC, marzo 2012, disponible en: http://tribunas.racc.es/mapa_vial_espanyol_en_los_ultimos_20_anyos

REE, Indicadores Nacionales. 2015. www.ree.es

REE, Gestor de la red y transportista. Planificación y desarrollo de la red. Acceso en octubre de 2015. Disponible en: http://www.ree.es/es/actividades/gestor-de-la-red-y-transportista/planificacion-y-desarrollode-la-red

REE, Informe del Sistema Eléctrico Español 2000, 2001. Disponible en: http:// www.ree.es/es/publicaciones/2013/10/informe-del-sistema-electrico-espanol-2000

REE, Informe del Sistema Eléctrico Español 2014, 2015. Disponible en: http:// www.ree.es/es/publicaciones/sistema-electrico-espanol/informe-anual/ informe-del-sistema-electrico-espanol-2014

REVISTA OIL\&GAS, Julio-agosto 2015.

SÁNCHEZ, R., "La gran autopista eléctrica de Alemania", El Mundo, (13/02/2014).

SEDIGAS, Informe anual 2014, 2015. Disponible en: http://www.sedigas.es/ pagina.php? $\mathrm{p}=11$

SERVICIO DE ESTUDIOS DE BANCO DE ESPAÑA, El Análisis de la Economía Española, ed. Alianza Editorial, Madrid, 2005.

SMIL, V., Energy Transitions: History, Requirements, Prospects, 2010.

STILWELL. M., EDP: Energía Sostenible y Competitiva. Deusto Forum, conferencia en Deusto Business School, septiembre 2015.

TERRASA, R., “PPor qué no arranca el coche eléctrico en España?”, El Mundo (2013), disponible en: http://www.elmundo.es/elmundo/2013/07/16/ valencia/1373980924.html

UNESA, Contribución de las Compañias que integran UNESA al Desarrollo de la Sociedad Española, 2013. 
Tres Retos PARA LA ENERGÍA EN ESPAÑA: COMPETITIVIDAD, SEGURIDAD Y CRECIMIENTO

VON HIRSCHHAUSEN, "The German "Energiewende". An introduction". Economics of Energy E Environmental Policy, 2014.

WEISS y otros, "On the electrification of road transport. Learning rates and prices forecasts for hybrid-electric and battery electric vehicles", Energy Policy, September 2012. 
